\title{
Transient Analysis of Crack in Composite Layered Medium Subjected to Dynamic Loadings
}

\author{
Chien-Ching Ma*and Yi-Shyong Ing ${ }^{\dagger}$ \\ National Taiwan University, Taipei 10617, Taiwan, Republic of China
}

\begin{abstract}
A semi-infinite crack in a layered medium subjected to antiplane dynamic loading is investigated. In analyzing this problem, the fact that the reflections and diffractions of stress waves by the interface boundary and by the crack will generate an infinite number of waves must be taken into account. A useful fundamental solution is proposed, and the solution is determined by superposition of the fundamental solution in the Laplace transform domain. The proposed fundamental problem is the application of exponentially distributed traction (in the Laplace transform domain) on the crack faces. Cagniard's method for Laplace inversion is used to obtain the transient solution in time domain. The final formulations for the stress intensity factor are expressed explicitly and the dynamic effect of each wave is presented in a closed form. The results are valid for an infinite number of waves that are scattered from the crack tip and reflected by the interface. Numerical calculations of dynamic stress intensity factors are evaluated and discussed in detail.
\end{abstract}

\section{Introduction}

$\mathbf{M}$ OST of the analyses done regarding cracked bodies are quasistatic. There are numerous situations in which the material inertia becomes significant and must be taken into account in the analyses. The question of whether or not inertial effects are significant depends on the loading conditions and the geometric configuration of the body. Inertial effects can arise either from applying dynamic loading on a cracked solid or from rapid crack propagation. The inherent time dependence of a dynamic fracture process results in mathematical models that are more complex than equivalent quasistatic models. However, there is substantial interest in the dynamic fracture problem because of its importance in many engineering applications. The problem is encountered in impact damage to fan blades and automotive and aircraft windshields.

The difficulty in determiningthe transient stress field in a crackedelastic body subjected to dynamic loading is well known. A preexisting crack inside the medium would disturb the propagation waves and make the theoretical analysis much more difficult than that in a homogeneous medium. A considerable amount of research has been directed toward the solution of problems involving the interaction of stress waves with the crack and boundary to improve understanding of the behavior of material failure under dynamic loading.

In conventional studies of a semi-infinite crack in an unbounded medium subjected to dynamic loading, the complete solution is obtained by integral transform methods together with direct application of the Wiener-Hopf technique ${ }^{1}$ and the Cagniard-de Hoop method $^{2}$ of Laplace transform inversion. If the loading is replaced by a nonuniform distribution having a characteristiclength, then the same procedure using integral transformation methods does not apply. The problem of an elastic solid containing a semi-infinite crack subjected to concentrated point loading on the faces of the crack has been studied by Freund. ${ }^{3}$ He proposed a fundamental solution arising from an edge dislocation climbing along the line ahead of the crack tip with a constant speed to overcome these difficulties of the case with a characteristiclength. The solution can be constructed by taking an integrationover a climbing dislocation of different moving velocity. Basing their procedure on this method, Brock ${ }^{4-6}$ and Ma and $\mathrm{Hou}^{7,8}$ have analyzed a series of problems of a semi-infinite crack subjected to impact loading. For the problem of a crack of finite length subjected to a suddenly applied crack face pressure,

Received Feb. 13, 1996; revision received July 25, 1996; accepted for publication Dec. 23, 1996. Copyright 997 by the American Institute of Aeronautics and Astronautics, Inc. All cights reserved.

$*$ Professor, Department of Mechanical Engineering.

${ }^{\dagger}$ Graduate Student, Department of Mechanical Engineering. the integral transforms can be applied and a relationship among sectionally analytical function is obtained that is somewhat more complicated in form than the standard Wiener-Hopf equations. In principal, the generalized Wiener-Hopf equations can be solved iteratively to obtain the complete solution. In practice, however, only the first step in the iteration process has been carried out by Thau and $\mathrm{Lu}^{9}$ up until a dilatational wave has traveled the length of the crack twice. A thorough summary of the application of the main direct methods of analysis for transient problems in dynamic fracture for elastic or inelastic problems has been given by Freund. ${ }^{10}$ For an incident antiplane harmonic wave, Sih and Loeber ${ }^{11}$ and $\mathrm{Mal}^{12}$ made detailed studies of the displacement and the stress field in the vicinity of a finite crack.

Most of the solved dynamic fracture problems are regarded as a semi-infinite crack subjected to a uniformly distributed dynamic loading on the crack faces or subjected to incident plane waves. For the problems mentioned earlier, either the direct application of the well-known Wiener-Hopf technique is used or the superposition method proposed by Freund is performed to solve the problems. However, if a crack is subjected to incident nonplanar waves, none of the known methods can be used to obtain the transient solutions. Recently Tsai and $\mathrm{Ma}^{13}$ proposed a new fundamental solution to overcome these difficulties. The fundamental problem they considered is an exponentially distributed traction applied on crack faces, and the solution is constructed by superposition of the fundamental solution in the Laplace transform domain. Tsai and $\mathrm{Ma}^{13}$ used this new fundamental solution to obtain the transient solution for a stationary semi-infinite crack subjected to a suddenly applied dynamic inplane body force in an unbounded medium. This alternative fundamental solution also is applied successfully toward solving more complicated transient problems ${ }^{14-16}$ for a subsurface stationary inclined crack subjected to dynamic loadings.

Determination of the dependence of the dynamic stress intensity factor (SIF) on body configuration and applied loading is a principal objective in dynamic fracture. Analytical results for configurations with boundaries other than the crack faces are rare. The interaction of stress waves with cracks provides a way of altering the cracktip stress field, and thus provide the basis for the crack-branching phenomenon.

Because the interaction of stress waves with a crack is an important event, we investigate it in greaterdetail. We extend the methodology that was used successfully in solving a crack in a homogeneous medium to construct the transient solution for a crack in the configuration of a layered medium. Consider a layer of elastic material with height $2 l$ that is sandwiched between two elastic half-spaces, as shown in Fig. 1. A semi-infinite crack lies in the midplane of the layer and is parallel to the interface. A dynamic concentrated line load is applied tangentially to the free surfaces of the semi-infinite 


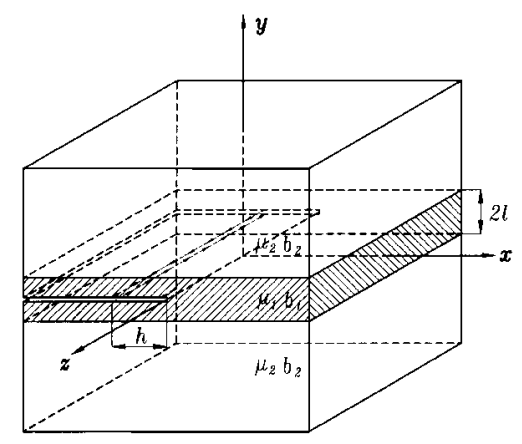

Fig. 1 Configuration and coordinate systems for a semi-infinite straight crack in a sandwiched layered medium.

\begin{tabular}{llll}
$\mu_{2} b_{2}$ & $\overbrace{\mu_{1} b_{t}}^{y}$ \\
\hline & $\rho_{2} b_{2}$ & $l$
\end{tabular}

Fig. 2 Configuration and coordinate systems of a crack in a layered medium for two-dimensional analysis.

crack along a line parallel to the $z$ axis. This problem generates a deformation only in the $z$ direction and is called the antiplane strain deformation, which can be viewed as a two-dimensional problem, as shown in Fig. 2. In analyzing the problems, the interface boundaries and crack are considered in the analysis. The interaction of the reflected waves generated from the interface boundaries and the crack must be taken into account, which will make the analysis extremely difficult. It is impossible to solve this complicated problem by using the standard Wiener-Hopf technique, and so, some other approach must be followed. A useful fundamental solution is proposed to overcome these difficulties. This proposed fundamental solution is successfully applied toward solving the problem and is demonstratedas an efficient methodologyto solve similar problems. Because the SIF is the key parameter in characterizing dynamic crack growth, we focus our attention mainly on the determination of the dynamic SIF.

\section{Required Fundamental Solutions}

In this study, we consider a semi-infinite straight crack in a layer with height $2 l$ of elastic medium that is sandwiched between two elastic half-spaces as shown in Fig. 1. A rectangular coordinate system labeled $x, y, z$ is introduced and the crack surface is in the $x-z$ plane. Dynamic concentrated line loads are applied uniformly along the upper and lower semi-infinite crack surfaces in the $z$ direction with a distance $h$ to the crack tip. The only nonzero component of displacement is in the $z$ direction and depends only on $x$ and $y$ coordinates, i.e., $u(x, y, z, t)=v(x, y, z, t)=0$ and $w(x, y, z, t)=w(x, y, t)$; such a deformation usually is called the antiplane deformation. Hence this problem can be simplified as a two-dimensional problem, as indicated in Fig. 2.

As usual in problems of the type considered here, superposition of solutions plays a significant role. The solutions of the problem considered in this study can be determined by superposition of the following problems. Problem A treats a dynamic concentrated loading acting on semi-infinite crack faces in an unbounded medium at time $t=0$; the incident wave will be diffracted from the crack tip and propagate toward the planes that will eventuallydefine the interface boundaries. In problem $\mathrm{B}$, an interface is considered in which the boundary is subjected to incident waves that are equal to the corresponding waves generated from the crack tip in problem A. Problem C considers an infinite body containing a semi-infinite crack in which the crack is subjected to the reflected waves that are generated by the interface boundary in problem $\mathrm{B}$. The three fundamental problems $\mathrm{A}, \mathrm{B}$, and $\mathrm{C}$ are superimposed to obtain the solution for stress waves interacting with a crack. The reflected waves induced from the interface boundary, as indicated in problem $\mathrm{B}$, can be obtained by constructing the solution for incident cylindricalwaves interacting with an interface in a layered medium. Problem A is a well-known problem for a semi-infinite crack in an unbounded medium, and many efforts have been devoted to analyzing this problem.

Problem $\mathrm{C}$ in the aforementioned three fundamental problems is the only one that needs careful analysis. The problem that we deal with is the interaction of a crack with cylindrical reflected waves, which causes the only difficulty in the analysis. For most of the dynamic problems, the propagating waves can be represented in an exponential functional form in the Laplace transform domain of time. The reflected and diffracted waves generated by the interface boundaries and by the crack thus can be constructed by the superposition method, that is, if the responses toward an applied exponentially distributed traction on boundaries in the Laplace transform domain can be obtained preliminarily. The proposed superposition scheme, unlike usual superposition methods that are performed in the time domain, is performed in the Laplace transform domain.

Consider the fundamental problem of antiplane deformation for a semi-infinite crack in an unbounded medium. The solution for an exponentially distributed loading applied at the crack faces in the Laplace transformdomain is referred to as the fundamentalsolution. The problem can be viewed as a half-planeproblem with the material occupying the region $y>0$ and subjected to the following mixed boundary conditions in the Laplace transform domain:

$$
\begin{array}{cc}
\bar{\tau}_{y z}(x, 0, s)=e^{s \eta x} & -\infty<x<0 \\
\bar{w}(x, 0, s)=0 & 0<x<\infty
\end{array}
$$

where $s$ is the variable of the Laplace transform on time $t$ and $\eta$ is a constant that can be considered as a superposition parameter. The overbar denotes the transform on time $t$. The one-sided Laplace transform with respect to time and the two-sided Laplace transform with respect to $x$ are defined by

$$
\begin{gathered}
\bar{w}(x, y, s)=\int_{0}^{\infty} w(x, y, t) e^{-s t} \mathrm{~d} t \\
\tilde{w}(\lambda, y, s)=\int_{-\infty}^{\infty} \bar{w}_{\bar{w}}(x, y, s) e^{-s \lambda x} \mathrm{~d} x
\end{gathered}
$$

where $\lambda$ is the variable of the two-sidedLaplace transformon $x$. This fundamentalproblem can be solved by using the standard transform method and the Wiener-Hopf technique. The governing equation can be represented by the two-dimensional wave equation

$$
\frac{\partial^{2} w}{\partial x^{2}}+\frac{\partial^{2} w}{\partial y^{2}}=b^{2} \frac{\partial^{2} w}{\partial t^{2}}
$$

where $b$ is the slowness of the shear wave given by

$$
b=1 / v_{s}=\sqrt{\rho / \mu}
$$

in which $w(x, y, t)$ is the displacement normal to the $x-y$ plane; $v_{s}$ is the shear wave speed, $\mu$ and $\rho$ are, respectively, the shear modulus and the mass density of the material. The nonvanishing shear stresses are

$$
\tau_{y z}=\mu \frac{\partial w}{\partial y}, \quad \tau_{x z}=\mu \frac{\partial w}{\partial x}
$$

This fundamental problem can be solved by the application of integral transforms. The one-sided Laplace transform is applied over time, the two-sided Laplace transform is applied over $x$ under the restriction of $\operatorname{Re}(\eta)>\operatorname{Re}(\lambda)$, and, finally, the Wiener-Hopf technique is implemented. The solutions of stresses and displacement expressed in the transform domain for the boundary conditions (1) and (2) are

$$
\begin{gathered}
\bar{\tau}_{y z}(x, y, s)=\frac{1}{2 \pi i} \int_{\Gamma_{\lambda}} \frac{\left.(b+\lambda)^{\frac{1}{2}} e^{-s(\alpha y}-\lambda x\right)}{(b+\eta)^{\frac{1}{2}}(\eta-\lambda)} \mathrm{d} \lambda \\
\bar{\tau}_{x z}(x, y, s)=-\frac{1}{2 \pi i} \int_{\Gamma_{\lambda}} \frac{\left.\lambda e^{-s(\alpha y}-\lambda x\right)}{(b+\eta)^{\frac{1}{2}}(\eta-\lambda)(b-\lambda)^{\frac{1}{2}}} \mathrm{~d} \lambda \\
\bar{w}(x, y, s)=-\frac{1}{2 \pi i} \int_{\Gamma_{\lambda}} \frac{\left.e^{-s(\alpha y}-\lambda x\right)}{\mu s(b+\eta)^{\frac{1}{2}}(\eta-\lambda)(b-\eta)^{\frac{1}{2}}} \mathrm{~d} \lambda
\end{gathered}
$$


where

$$
\alpha(\lambda)=\overline{b_{j}+\lambda} \overline{b_{j}-\lambda}=\alpha_{+}(\lambda) \alpha_{-}(\lambda)
$$

The corresponding result for the dynamic SIF in the Laplace transform domain is

$$
\begin{aligned}
\bar{K}(s) & =\lim _{x \rightarrow 0} \sqrt{2 \pi x} \bar{\tau}_{y z}(x, 0, s) \\
& =-\frac{\overline{2}}{\bar{s}\left(b \sqrt{-\eta)^{\frac{1}{2}}}\right.}
\end{aligned}
$$

\section{Transient Analysis of Dynamic SIF}

Consider the problem of a semi-infinite crack in an infinitely long strip of a layered medium, as shown in Fig. 2. At time $t=0$, a pair of concentrated antiplane dynamic loadings with magnitude $p$ are applied at the crack faces with a distance $h$ from the crack tip. The time dependence of the dynamic loading is represented by the Heaviside step function $H(t)$. In this problem, the cylindrical waves induced from the dynamic concentrated loading and the diffracted waves generated from the crack tip will be reflected from horizontal interface boundaries; these waves will interact with the crack at some later time. The major difficulty in analyzing this problem is the one that deals with the interaction of reflected waves with the semi-infinite crack, and the superposition technique of the fundamental solutions in the Laplace transform domain are used in the analysis. The analytical work provided in this study is valid for the case of $b_{1}<b_{2}$. The transient solutions are composed of incident field, reflected field, and diffracted field, which are denoted by superscripts of $i, r$, and $d$, respectively. Before the time that the $i$ and $d$ waves are reflected from the interface boundaries of the strip, the problem can be considered as a semi-infinite crack in an unbounded medium.

The incident field of the cylindrical wave generated by the concentrated loading expressed in the Laplace transform domain can be obtained as follows:

$$
\bar{\tau}_{y z}(x, 0, s)=\frac{1}{2 \pi i} \int_{\Gamma_{\lambda}}-p e^{s \lambda(x+h)} \mathrm{d} \lambda
$$

The applied traction on the crack face as indicated in Eq. (9) has a functional form $e^{s \lambda x}$. Because the solutions of applying traction $e^{s \eta x}$ on crack faces in the Laplace transform domain were solved in Sec. II, the diffracted field generated from the stationary semiinfinite crack can be constructedby superimposingthe incident wave traction that is equal to Eq. (9). When we combine Eqs. (5) and (9), the solution for a diffracted wave in the Laplace transform domain can be expressed as follows: where

$$
\begin{gathered}
\eta_{\Gamma}^{+}=-\frac{t_{1}}{h} \pm^{i \varepsilon,} \quad \eta_{\frac{1}{+}}=-\frac{t_{2}}{r_{2}} \cos \theta_{2} \pm^{i}\left(\frac{t_{2}^{2}}{r_{2}^{2}}-b_{1}^{2}\right)^{\frac{1}{2}} \sin \theta_{2} \\
\left.r=\left[(x+h)^{2}+y^{2}\right)\right]^{\frac{1}{2}}, \quad \theta=\cos ^{-1}\left(\frac{x+h}{r}\right) \\
r_{2}=\left(x^{2}+y^{2}\right)^{\frac{1}{2}}, \quad \theta_{2}=\cos ^{-1}\left(\frac{x}{r_{2}}\right) \\
H\left(\eta_{1}, \eta_{2}\right)=\frac{\left(b_{1}+\eta_{2}\right)^{\frac{1}{2}}}{\left(\eta_{1}-\eta_{2}\right)\left(b_{1}+\eta_{1}\right)^{\frac{1}{2}}} \quad t=t_{1}+t_{2}
\end{gathered}
$$
is

The corresponding dynamic SIF induced by the diffracted wave

$$
K^{s}(t)=p \sqrt{(2 / \pi h)} H\left(t-b_{1} h\right)
$$

The dynamic SIF shown in Eq. (13) jumps from zero at the instant that the cylindrical wave generated by the concentrated loading reaches the crack tip. This solution is the same as that for a semiinfinite crack in an unbounded medium and subjected to a dynamic point loading, which is also the equilibrium SIF for the specified applied loading on a semi-infinite crack.

After some later time, the incident wave ( $i$ wave) generated from the dynamic concentrated loading and the diffracted wave ( $d$ wave) radiated out from the crack tip will be reflected from the interface, which are indicated as the $i r$ and $d r$ waves, respectively. The solutions for reflected waves generated from the interface expressed in the Laplace transform domain can be obtained as follows:

$$
\begin{aligned}
& \bar{\tau}_{y z}^{i r+d r}(x, y, s)=\frac{1}{4 \pi^{2}} \\
& \quad \times \iint \frac{\gamma_{\frac{1}{2}}\left(\eta_{2}\right) p \alpha_{+}\left(\eta_{2}\right)}{\left(\eta_{1}-\eta_{2}\right) \alpha_{4}\left(\eta_{1}\right)} e^{s h \eta_{1}} \exp \left[s \alpha_{1}(y-2 l)+s \eta_{2} x\right] \mathrm{d} \eta_{2} \mathrm{~d} \eta_{1}
\end{aligned}
$$

where

$$
\gamma_{\frac{1}{2}}=\frac{\mu_{1} \alpha_{1}-\mu_{2} \alpha_{2}}{\mu_{1} \alpha_{1}+\mu_{2} \alpha_{2}}
$$

The induced dynamic SIF by $i r$ and $d r$ waves can be obtained by setting $y=0$ in Eq. (14) and superimposing the fundamental solution expressed in Eq. (8). The result for the SIFs expressed in the Laplace transform domain have the following form:

$$
\begin{aligned}
& \bar{K}^{i r d+d r d}(s)=\frac{-1}{4 \pi^{2}} \\
& \quad \times \iint \frac{p \overline{2} \gamma_{\frac{1}{2}}\left(\eta_{2}\right)}{\bar{s}\left(\eta_{1} \underline{-} \eta_{2}\right) \alpha_{4}\left(\eta_{1}\right)} \exp \left[s h \eta_{1}-2 s \alpha_{1}\left(\eta_{2}\right) l\right] \mathrm{d} \eta_{2} \mathrm{~d} \eta_{1}
\end{aligned}
$$

$$
\bar{\tau}_{y z}^{s}(x, y, s)=\frac{1}{2 \pi i} \int_{\Gamma_{\lambda}}-p\left[\frac{1}{2 \pi i} \int_{\Gamma_{\eta_{2}}} \frac{\left(b_{1}+\eta_{2}\right)^{\frac{1}{2}}}{\left(\lambda-\eta_{2}\right)\left(b_{1}+\lambda\right)^{\frac{1}{2}}} e^{s \lambda t} \exp \left[-s\left(\alpha_{1} y-\eta_{2} x\right)\right] \mathrm{d} \eta_{2}\right] \mathrm{d} \lambda
$$

By using the Cagniard-de Hoop method of Laplace inversion, the incident and diffracted stress fields in the time domain are obtained as follows:

$$
\tau_{y z}^{i}(x, y, t)=\frac{-p t \sin \theta}{\pi r\left(t^{2}-b_{1}^{2} r^{2}\right)^{\frac{1}{2}}} H\left(t-b_{1} r\right)
$$

$$
\begin{aligned}
& \tau_{y z}^{d}(x, y, t)=\frac{p}{2 \pi^{2}} \\
& \times \int_{b_{1} h}^{{ }^{t}-b_{1} r_{2}} \operatorname{Re}\left[H\left(\eta_{1}^{+}, \eta_{2}^{+}\right) \frac{\partial \eta_{1}^{+}}{\partial t_{1}} \frac{\partial \eta_{2}^{+}}{\partial t_{2}}-H\left(\eta_{\overline{1}}, \eta_{2}^{+}\right) \frac{\partial \eta_{1}}{\partial t_{1}} \frac{\partial \eta_{2}^{+}}{\partial t_{2}}\right] \mathrm{d} t_{1}
\end{aligned}
$$

The dynamic SIFs expressed in time domain are

$$
K^{i r d}(t)=\frac{\overline{2} p}{\lambda^{\frac{3}{2}}} \int_{b_{1} r_{1}}^{t} \frac{1}{\overline{t^{-\tau}}} \operatorname{Im}\left[\frac{\gamma_{\frac{1}{2}}\left(\lambda^{+}\right)\left(\partial \lambda^{+} / \partial t\right)}{\alpha_{+}\left(\lambda^{+}\right)}\right]_{t=\tau} \mathrm{d} \tau
$$

where

$$
\begin{gathered}
\lambda^{+}=\frac{t_{1} \cos \theta_{1}}{r_{1}}+i \frac{\sin \theta_{1}}{r_{1}} \sqrt{i_{1}^{2}-b_{1}^{2} r_{1}^{2}} \\
r_{1}=\left[h^{2}+(2 l)^{2}\right]^{\frac{1}{2}}, \quad \theta_{1}=\cos ^{-1}\left(\frac{-h}{r_{1}}\right)
\end{gathered}
$$


and

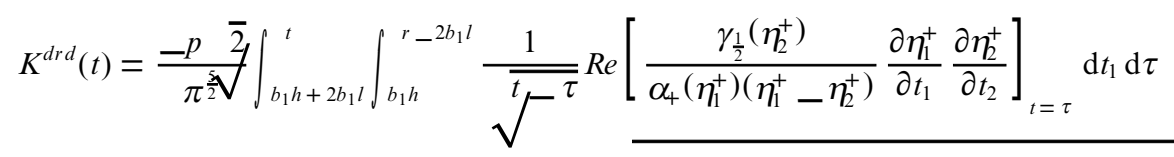

where

$$
\eta_{+}^{+}=-\left(t_{1} / h\right) \pm^{i \varepsilon,} \quad \eta_{\frac{2}{+}}= \pm i \sqrt{\left.t_{2}^{2} / 4 l^{2}\right)-b_{1}^{2}}, \quad t_{1}+t_{2}=t
$$

Finally, the complete transient solutions for dynamic SIF that account for the contributions of all the reflected and diffracted waves are obtained explicitly. The solutions can be simplified into a very compact form as follows:

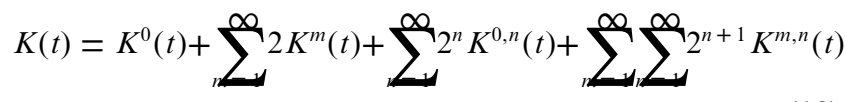

where

$$
\begin{gathered}
K^{0}(t)=p \sqrt{(2 / \pi h)} H\left(t-b_{1} h\right) \\
K^{m}(t)=\frac{p \overline{2}}{\pi \sqrt[3]{2}} \int_{b_{1} r_{m}}^{t} \frac{1}{\bar{t}^{-\tau}} \operatorname{Im}\left\{\frac{\left[\gamma_{\frac{1}{2}}\left(\eta_{1, m}^{+}\right)^{m}\right.}{\alpha_{+}\left(\eta_{1, m}^{+}\right)} \frac{\partial \eta_{1, m}^{+}}{\partial t_{1}}\right\}_{t_{1}=\tau} \mathrm{d} \tau
\end{gathered}
$$

Note that the solutions for more general crack face loadings can be obtained on the basis of the result shown in Eq. (18). Suppose that the crack faces are loaded by a pair of concentrated dynamic loadings applied at $x=\lfloor h$ with time dependent $p g(t)$ and $g(t)=0$ for $t<0$. The dynamic SIF for this modified problem is

$$
K^{g}(t)=\int_{0}^{t} K(t-\xi) \dot{g}(\xi) \mathrm{d} \xi
$$

However, if a traction distribution $p f(x)$ is suddenly applied over the interval $x_{1}<x<x_{2}$ on crack faces, then the transient SIF of the problem is

$$
K^{f}(t)=\int_{x_{1}}^{x_{2}} K(t ; x) f(x) \mathrm{d} x
$$

where $K(t)$ has been obtained explicitly in Eq. (18).

For the numerical calculation of the transient SIF, we consider a semi-infinite crack in a layered medium and subjected to a pair of dynamic concentrated loadings on the stationary crack faces at time $t=0$. The induced wave fronts for reflected, refracted, and diffracted in a short time period are plotted in Fig. 3. The

$$
K^{m, n}(t)=\frac{2 p \overline{2}^{q}}{\bar{\pi}(2 \lambda i)^{n+1}} \int_{b_{1} r_{m}+2 n b_{1} l}^{t} \int_{b_{1} r_{m}}^{a_{1}} \int_{2 b_{1} l}^{a_{2}} \int_{2 b_{1} l}^{a_{3}} \ldots \int_{2 b_{1} l}^{a_{n}} \frac{1}{\sqrt{t}-\tau} \operatorname{SIF}_{m, n} \mathrm{~d} t_{n} \mathrm{~d} t_{n}-1 \quad \ldots \mathrm{d} t_{1} \mathrm{~d} \tau
$$

in which

$$
\begin{aligned}
& a_{1}=\tau-2 n b_{1} l \\
& a_{j}=\tau-t_{1}-t_{2}-\ldots-t_{j-1}-2(n-j+1) b_{1} l \\
& q=0 \quad \text { when } \quad n=1,3,5, \ldots \\
& q=1 \quad \text { when } n=2,4,6, \ldots \\
& \eta_{\mathbb{1}, m}^{+}=\frac{t_{1} \cos \theta_{m}}{r_{m}} \pm i \frac{\sin \theta_{m}}{r_{m}} \sqrt{t_{1}^{2}-b_{1}^{2} r_{m}^{2}} \\
& r_{m}=\left[h^{2}+(2 m l)^{2}\right]^{\frac{1}{2}} ; \quad \theta_{m}=\cos ^{-1}\left(\frac{-h}{r_{m}}\right) \\
& \eta_{J}^{ \pm}= \pm \sqrt{\left(\frac{t_{j}^{2}}{4 l^{2}}\right)-b_{1}^{2}}, \quad j=2,3,4, \ldots \\
& t_{j}=t-t_{1}-t_{2}-\ldots-t_{j_{-} 1}, \quad j=2,3,4, \ldots \\
& \gamma_{\frac{1}{2}}(\eta)=\frac{\mu_{1} \alpha_{1}(\eta)-\mu_{2} \alpha_{2}(\eta)}{\mu_{1} \alpha_{1}(\eta)+\mu_{2} \alpha_{2}(\eta)} \\
& \alpha_{1}(\eta)=\alpha_{4}(\eta) \alpha \underline{(}(\eta)=\sqrt{b_{1}+\eta} \sqrt{b_{1}-\eta} \\
& \alpha_{2}(\eta)=\sqrt{b_{2}+\eta} \sqrt{b_{2}-\eta}
\end{aligned}
$$$$
j=2,3, \ldots, n
$$

time dependence of the dynamic loading is the Heaviside function $H(t)$. The dynamic SIFs for various situations are shown in Figs. 4-9. Figure 4 shows the dynamic SIF for $\mu_{2} / \mu_{1}=0$, which is the case for a semi-infinite crack in an infinitely long strip with traction-free boundary conditions on strip surfaces. We can see that the transient solution approaches the corresponding static value $\left(K=p \quad(2 / l)\left[1 \_e^{-\pi h / l}\right]^{-1 / 2}\right)$ after the first few waves have passed the crackt p, and the low $h / l$ ratio case approaches the static value faster than the high $h / l$ ratio case. Figures 5-7 show the transient response of dynamic SIF for different values of $\mu_{2} / \mu_{1}$ for $h / l=0.5$,

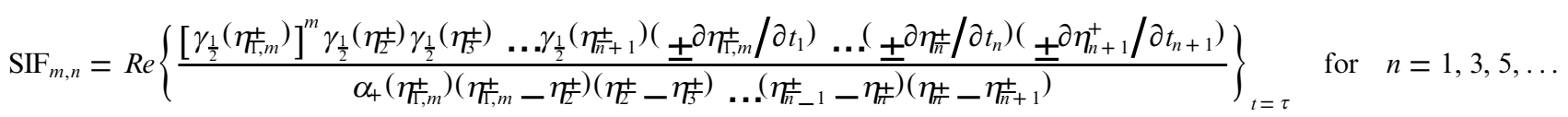

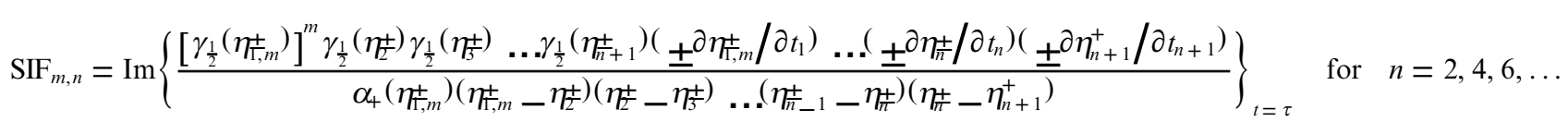

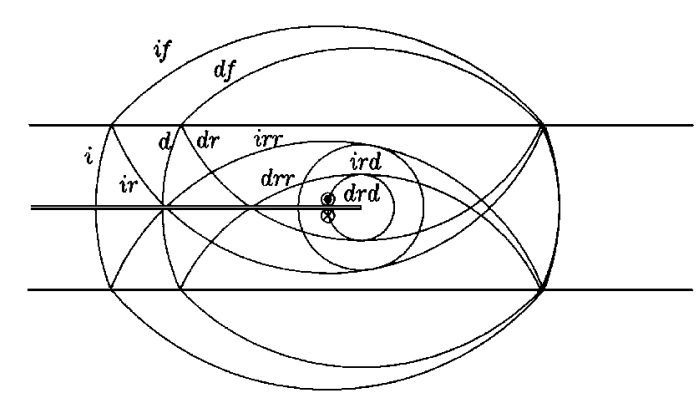

Fig. 3 Configuration of wave fronts in the layered medium during a short time period. 


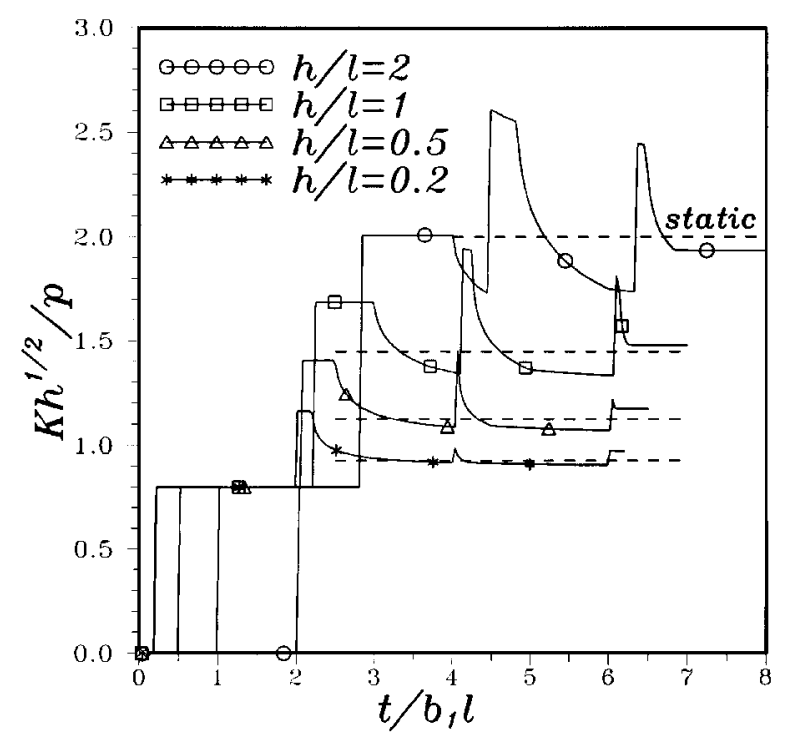

Fig. 4 Transient response of the dynamic SIF for traction-free boundary condition $\mu_{2} l \mu_{1}=\mathbf{0}$.

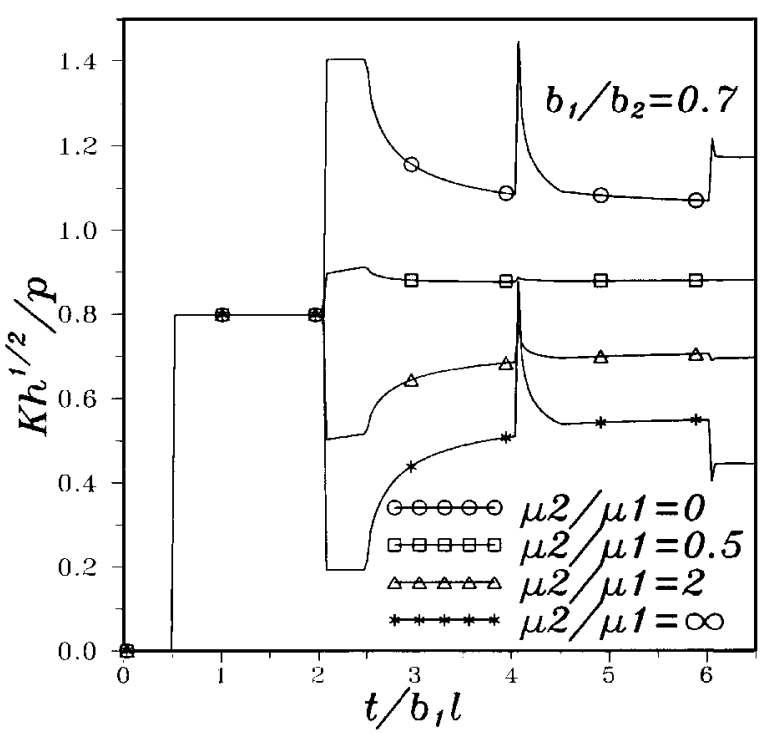

Fig. 5 Transient response of the dynamic SIF for different ratio of shear modulus for $h / l=0.5$.

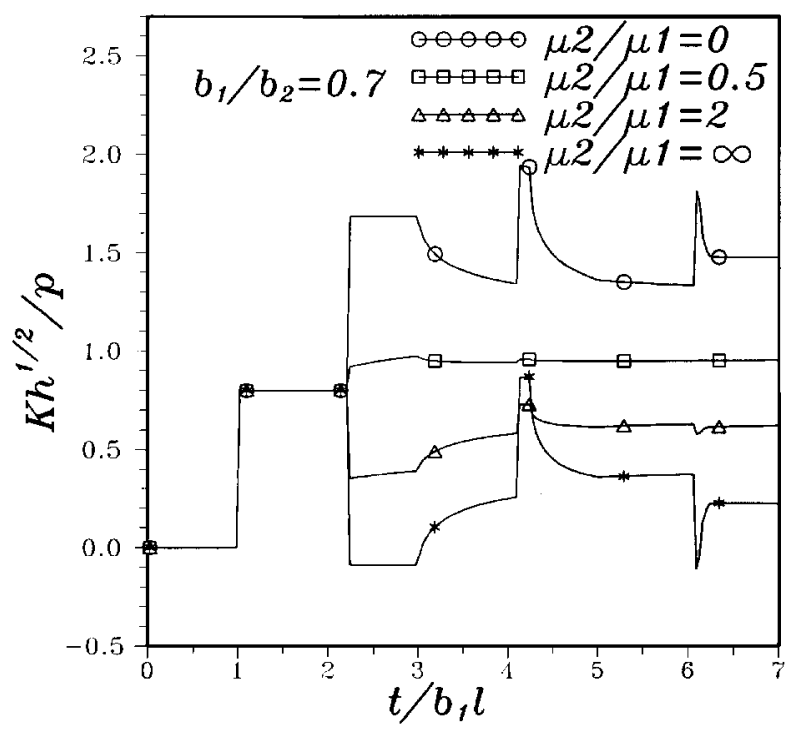

Fig. 6 Transient response of the dynamic SIF for different ratio of shear modulus for $h l l=1$.

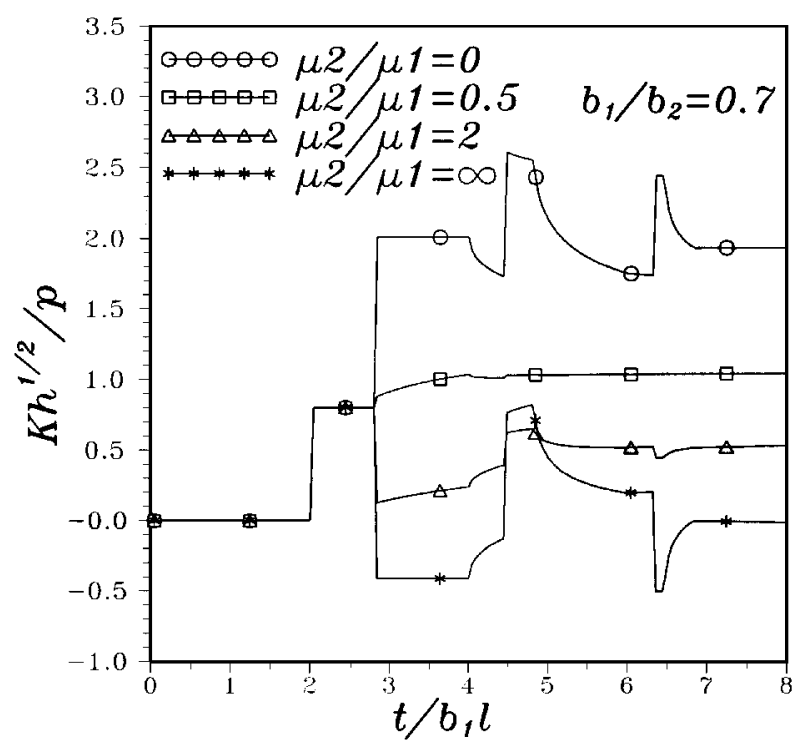

Fig. 7 Transient response of the dynamic SIF for different ratio of shear modulus for $h / I=2$.

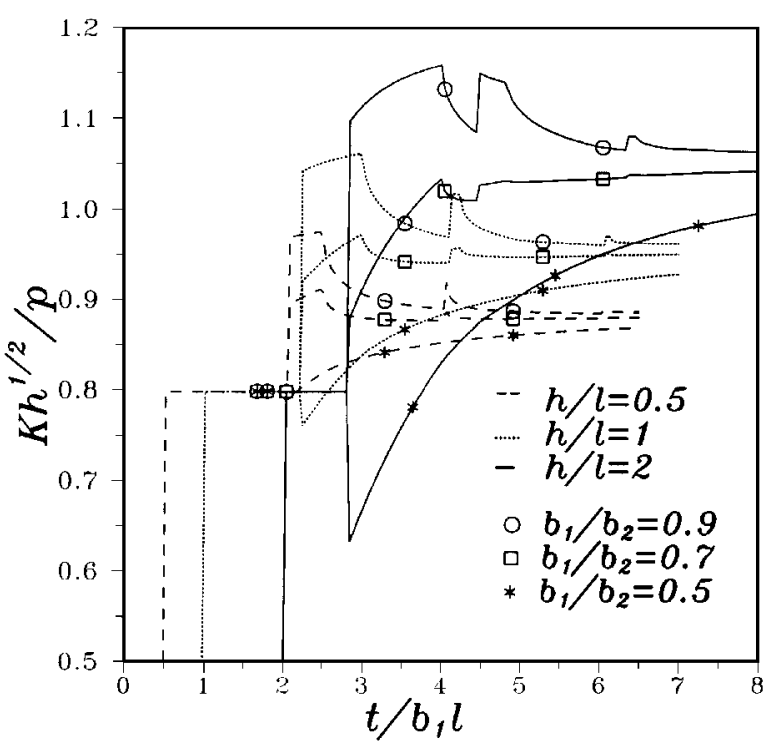

Fig. 8 Transient response of the dynamic SIF for different ratio of shear wave speed for $\mu_{2} l \mu_{1}=0.5$.

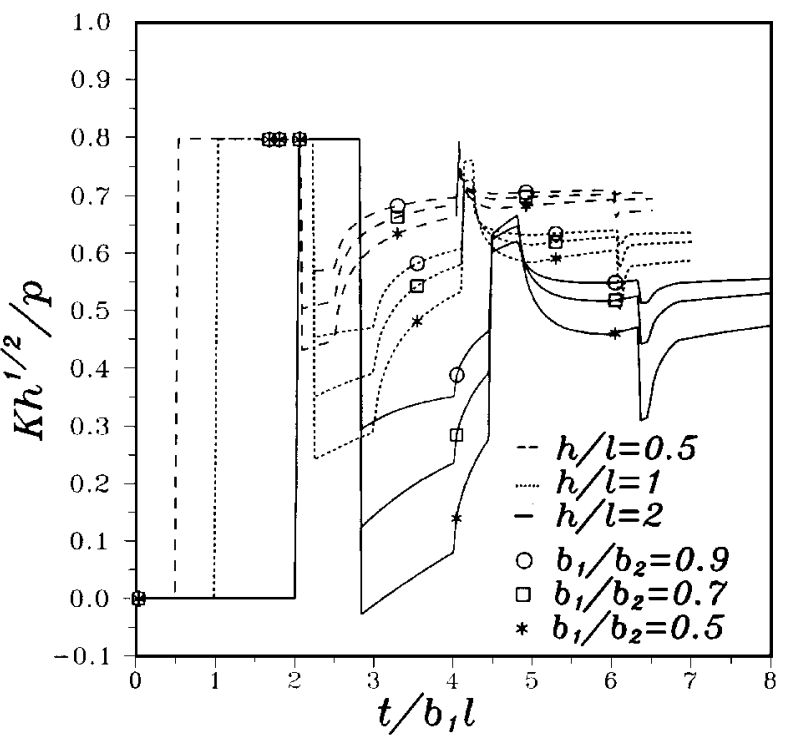

Fig. 9 Transient response of the dynamic SIF for different ratio of shear wave speed for $\mu_{2} l \mu_{1}=2$. 
1 , and 2 , respectively. The case for $\mu_{2} / \mu_{1}=0$ corresponds to the traction-free boundary condition, and $\mu_{2} / \mu_{1}=\infty$ corresponds to the rigid boundary conditions. It is indicated that the material properties of the layered medium affect the transient behavior quite significantly and $\mu_{2} / \mu_{1}=0$ cause the highest value of SIF whereas $\mu_{2} / \mu_{1}=\infty$ induces the lowest value. Figures 8 and 9 represent the results for different ratios of shear wave speed $b_{1} / b_{2}$ and $h / l$. Note that the lower $\mu_{2} / \mu_{1}$ ratio always causes the higher SIF, and the traction-free boundary condition (i.e., $\mu_{2} / \mu_{1}=0$ ) induces the largest value of SIF during the transient period.

\section{Conclusions}

The phenomena of crack propagation, arrest, and branching are important subjects in the areas of dynamic fracture analysis. The interaction of reflected waves with a crack for dynamic loading situations had been discussed only in experimentalworks. Experimental results indicated that the reflected waves dominate the stability of crack propagation. It is very important to have the analytical results to investigate this important event, but it seems difficult to obtain the analyticalsolutions by using the well-known conventional method.

We propose a powerful superposition methodology, and a useful fundamental problem is constructed. The fundamental problem is that of applying an exponentially distributed traction on the crack face, and the transient solution is determined by superposition of the fundamental solution in the Laplace transform domain. A pair of dynamic loadings applied on semi-infinite crack faces in a configuration of layered medium with a strip is investigated. An explicit and completeresult for the dynamic SIF is obtained in a closed form, and numerical results are evaluated in detail. The numerical results show that the SIFs induced by reflected waves from the interface are significant and the traction-free boundary will cause the highest SIF in the transient analysis.

In the foregoing sections, the dependence of the transient history of the SIF on the applied loading and geometry is shown. The SIF provides a one-parameter representation of the stress level near the crack tip and provides a basis for quantifying the resistance of materials to the onset of growth of a preexisting crack. There still are many unanswered questions in dynamic fracture, and this work may provide a useful technique for further investigation in more complicated dynamic fracture problems especiallyon the crack propagation event. The proposed method already has been extended to solve the more difficult problem of crack propagation with boundary effects, and the results will be shown in a future paper.

\section{Acknowledgment}

The authors gratefully acknowledge the financial support of this research by the National Science Council, Taiwan, Republic of China, under Grant NSC 83-0401-E-002-115.

\section{References}

${ }^{1}$ Noble, B., The Wiener-Hopf Technique, Pergamon, New York, 1958, Chap. 1.

${ }^{2}$ De Hoop, A. T., "Representation Theorems for the Displacement in an Elastic Solid and Their Application to Elastodynamic Diffraction Theory," Ph.D. Dissertation, Technische Hoegschool, Delft, The Netherlands, 1958.

${ }^{3}$ Freund, L. B., "The Stress Intensity Factor Due to Normal Impact Loading of the Faces of a Crack," International Journal of Engineering Science, Vol. 12, No. 1, 1974, pp. 179-189.

${ }^{4}$ Brock, L. M., "Shear and Normal Impact Loadings on One Face of a Narrow Slit," International Journal of Solids and Structures, Vol. 18, No. 6, 1982, pp. 467-477.

${ }^{5}$ Brock, L. M., "Stresses in a Surface Obstacle Undercut Due to Rapid Indentation," Journal of Elasticity, Vol. 14, No. 4, 1984, pp. 415-424.

${ }^{6}$ Brock, L. M., Jolles, M., and Schroedl, M., "Dynamic Impact over a Subsurface Crack: Applications to the Dynamic Tear Test," Journal of Applied Mechanics, Vol. 52, No. 2, 1985, pp. 287-290.

${ }^{7}$ Ma, C. C., and Hou, Y. C., "Theoretical Analysis of the Transient Response for a Stationary Inplane Crack Subjected to Dynamic Impact Loading," International Journal of Engineering Science, Vol. 28, No. 12, 1990, pp. 1321-1329.

${ }^{8}$ Ma, C. C., and Hou, Y. C., "Transient Analysis for Antiplane Crack Subjected to Dynamic Loadings," Journal of Applied Mechanics, Vol. 58, No. 3, 1991, pp. 703-709.

${ }^{9}$ Thau, S. A., and Lu, T. H., "Transient Stress Intensity Factors for a Finite Crack in an Elastic Solid Caused by a Dilatational Wave," International Journal of Solids and Structures, Vol. 7, No. 7, 1971, pp. 731750 .

${ }^{10}$ Freund, L. B., Dynamic Fracture Mechanics, Cambridge Univ. Press, Cambridge, England, UK, 1990, Chap. 6.

${ }^{11}$ Sih, G. C., and Loeber, J. F., "Wave Propagation in an Elastic Solid with a Line of Discontinuity or Finite Crack," Quarterly of Applied Mathematics, Vol. 27, No. 2, 1969, pp. 193-213.

${ }^{12}$ Mal, A. K., "Interaction of Elastic Waves with a Griffith Crack," International Journal of Engineering Science, Vol. 8, 1970, pp. 763-776.

${ }^{13}$ Tsai, C. H., and Ma, C. C., "Transient Analysis of a Semi-Infinite Crack Subjected to Dynamic Concentrated Forces," Journal of Applied Mechanics, Vol. 59, No. 4, 1992, pp. 804-811.

${ }^{14}$ Tsai, C. H., and Ma, C. C., "The Transient Analysis of a Subsurface Inclined Crack Subjected to a Buried Dynamic Dilatational Source," International Journal of Solids and Structures, Vol. 30, No. 3, 1993, pp. 399 412.

${ }^{15}$ Tsai, C. H., and Ma, C. C., "The Stress Intensity Factor of a Subsurface Inclined Crack Subjected to a Dynamic Impact Loading," International Journal of Solids and Structures, Vol. 30, No. 16, 1993, pp. 2163 2175.

${ }^{16}$ Ma, C. C., and Chen, S. K., "Exact Transient Full Field Analysis of an Antiplane Subsurface Crack Subjected to Dynamic Impact Loading," Journal of Applied Mechanics, Vol. 61, No. 3, 1994, pp. 649-655.

R. K. Kapania Associate Editor 